\title{
COVID-19 - A Review of the Impact it has made on Supportive and Palliative Care Services Within a Tertiary Hospital and Cancer Centre in Singapore
}

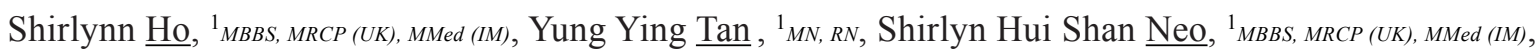
Qingyuan Zhuang, ${ }^{1}$ MBBS, MMED (FM), Min Chiam, ${ }^{2}$ MSc (Med Hum), Jamie Xuelian Zhou, $1,3,4{ }_{M B B S, ~ M R C P}(\mathrm{UK})$, MMed (IM), Natalie Liling Woong, ${ }^{5}{ }_{M B B S}$, Guozhang $\underline{\text { Lee }},{ }^{5}{ }_{M B B S, M R C P}(\mathrm{UK})$, MMed (IM), Lalit Kumar Radha Krishna, ${ }^{1,2,6-8}{ }_{M B C h B, F R C P, F A M S}$

\section{Introduction}

Singapore experienced its first case of COVID-19 on 23 January 2020 and within 2 weeks, the Disease Outbreak Response System Condition (DORSCON) was elevated from yellow to orange to escalate and guide national response. ${ }^{1,2}$ In order to halt community spread of the infection, which had gained momentum in the course of 2 months, Singapore introduced the 'circuit breaker' on 7 April 2020. This saw movement and interactions in public and private places restricted, with schools closing, travel and gatherings limited, and work-from-home measures instituted., ${ }^{3,4}$

The COVID-19 virus and the extraordinary measures set in place led to significant changes in the healthcare landscape and the delivery of Supportive and Palliative Care (SPC) services in Singapore. To meet these challenges, the Division of SPC (DSPC) at National Cancer Centre Singapore (NCCS) had to adapt its in-patient consult service to the oncology, haematology, renal, respiratory and Intensive Care Units (ICU) at Singapore General Hospital (SGH) and its own outpatient SPC services.

Our applied strategies, their impact and lessons learnt are featured in Table 1. Here, we focus on the key learning points that may offer guidance to other healthcare professionals (HCP)s facing similar problems brought on by the crisis.

\section{Inpatient service}

In light of the pandemic, consultant led inpatient services saw 3 significant changes to its practice.
First, to ensure social distancing, joint ward rounds and in-person multidisciplinary team meetings (MDM) s with haematology, oncology and ICU teams were suspended. In their place, small segregated autonomous teams, each consisting of a specialist nurse, medical officer and a senior clinician (henceforth "small teams"), were formed. These small teams functioned within specific geographical locations within the campus and were an adaption to redeployments of HCPs to specialised COVID-19 wards, and screening areas on and off-site. To circumvent the shortage of SPC presence on many wards, the small teams established symptom control guidelines, provided telephone advice and made palliative care resources available online. ${ }^{5}$

Secondly, in pre-empting the overwhelming of the healthcare system, focus was placed on discharge and advanced care planning conversations whenever possible. Although symptom management was not at risk, manpower limitations raised concerns over the compromise of holistic support provided to sickly patients and families that were physically separated from one another. Indeed, whilst DSPC saw a $22.2 \%$ reduction in referrals, the proportion of complex clinical and psychosocial referrals increased. As such, medical social workers (MSWs) from NCCS were called to participate in online case discussions and MDMs, and to provide telephone and teleconferencing support for patients and families who were facing difficulties with bereavement and grief.

Thirdly, in preparing for potential disruptions to supply chains and a surge in COVID-19 related ICU

\footnotetext{
${ }^{1}$ Division of Supportive and Palliative Care, National Cancer Centre Singapore, Singapore

${ }^{2}$ Division of Cancer Education, National Cancer Centre Singapore, Singapore

${ }^{3}$ Academic Clinical Programme, SingHealth Duke-NUS Academic Medical Centre, Singapore

${ }^{4}$ Lien Center for Palliative Care, Duke-NUS Graduate Medical School, Singapore

${ }^{5}$ Department of Internal Medicine, Singapore General Hospital, Singapore

${ }^{6}$ Palliative Care Institute Liverpool, Academic Palliative and End of Life Care Centre, University of Liverpool, United Kingdom; Cancer Research Centre,

University of Liverpool, United Kingdom

${ }^{7}$ Centre for Biomedical Ethics, National University of Singapore, Singapore

${ }^{8} \mathrm{PalC}$, The Palliative Care Centre for Excellence in Research and Education, Singapore

Address for Correspondence: Dr Lalit Kumar Radha Krishna, Palliative Care Institute Liverpool, Academic Palliative and End of Life Care Centre, University of Liverpool, United Kingdom; Cancer Research Centre, University of Liverpool, 200 London Rd, Liverpool L3 9TA, United Kingdom

Email: lalit.radha-krishna@liverpool.ac.uk
} 


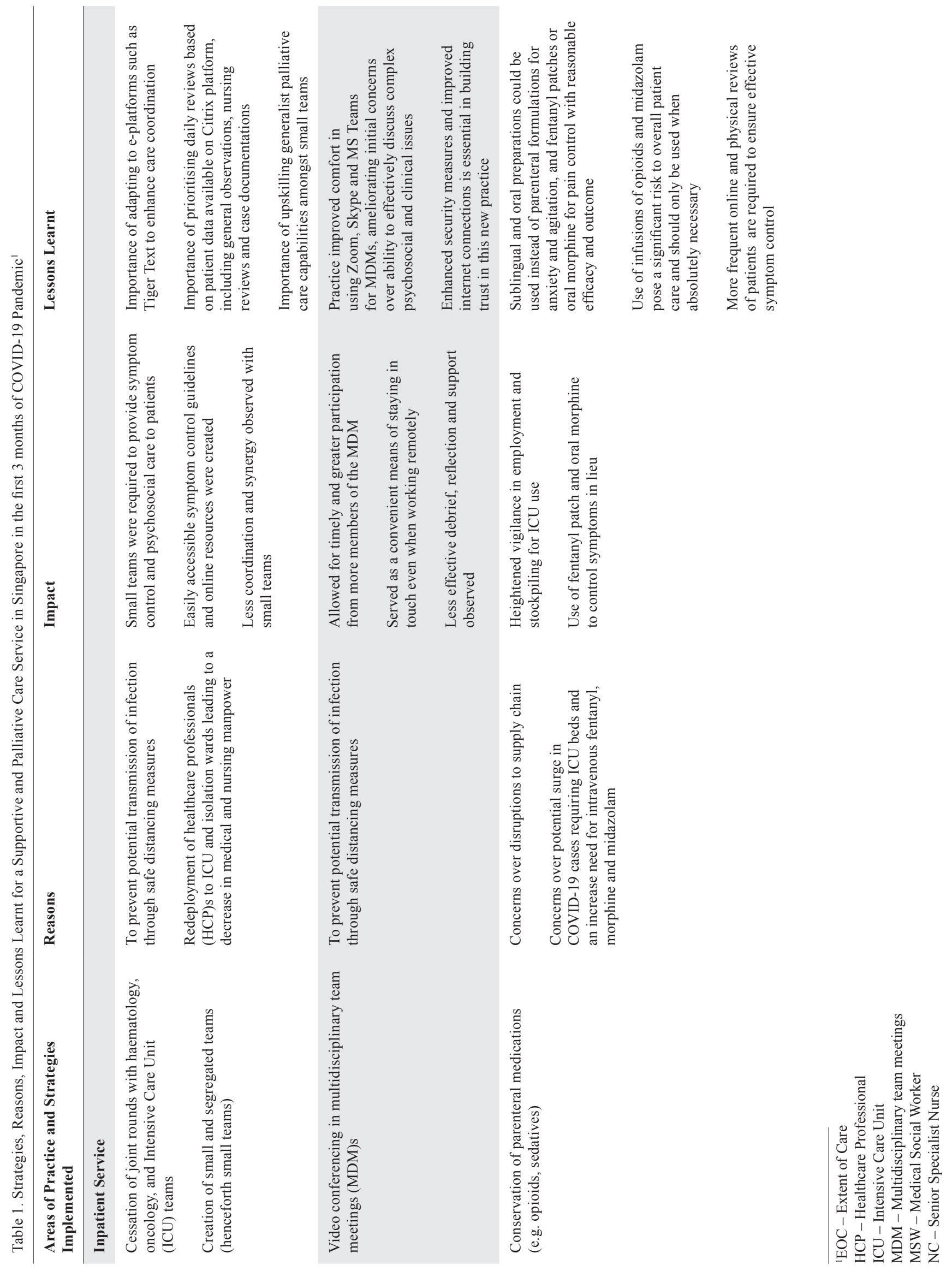




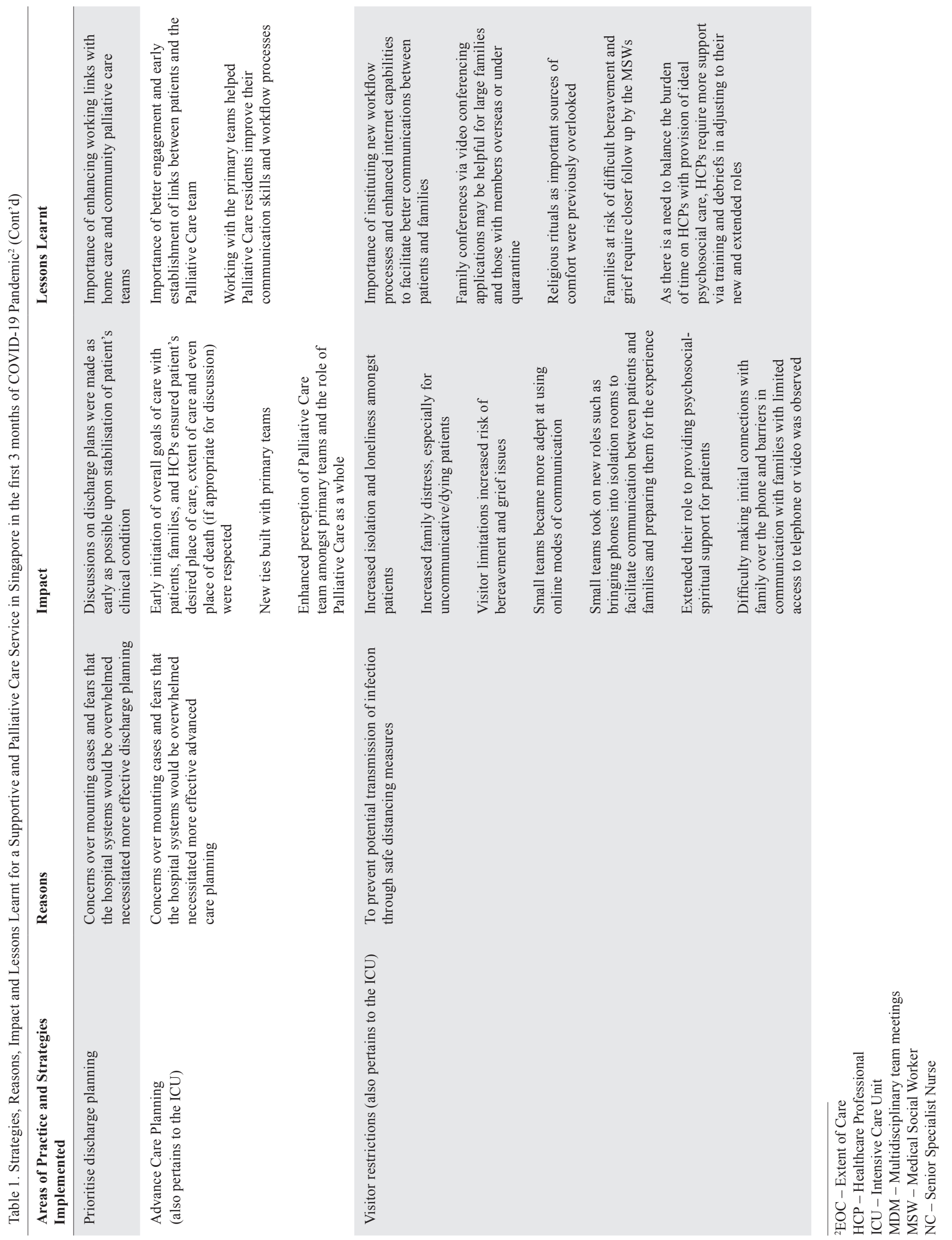




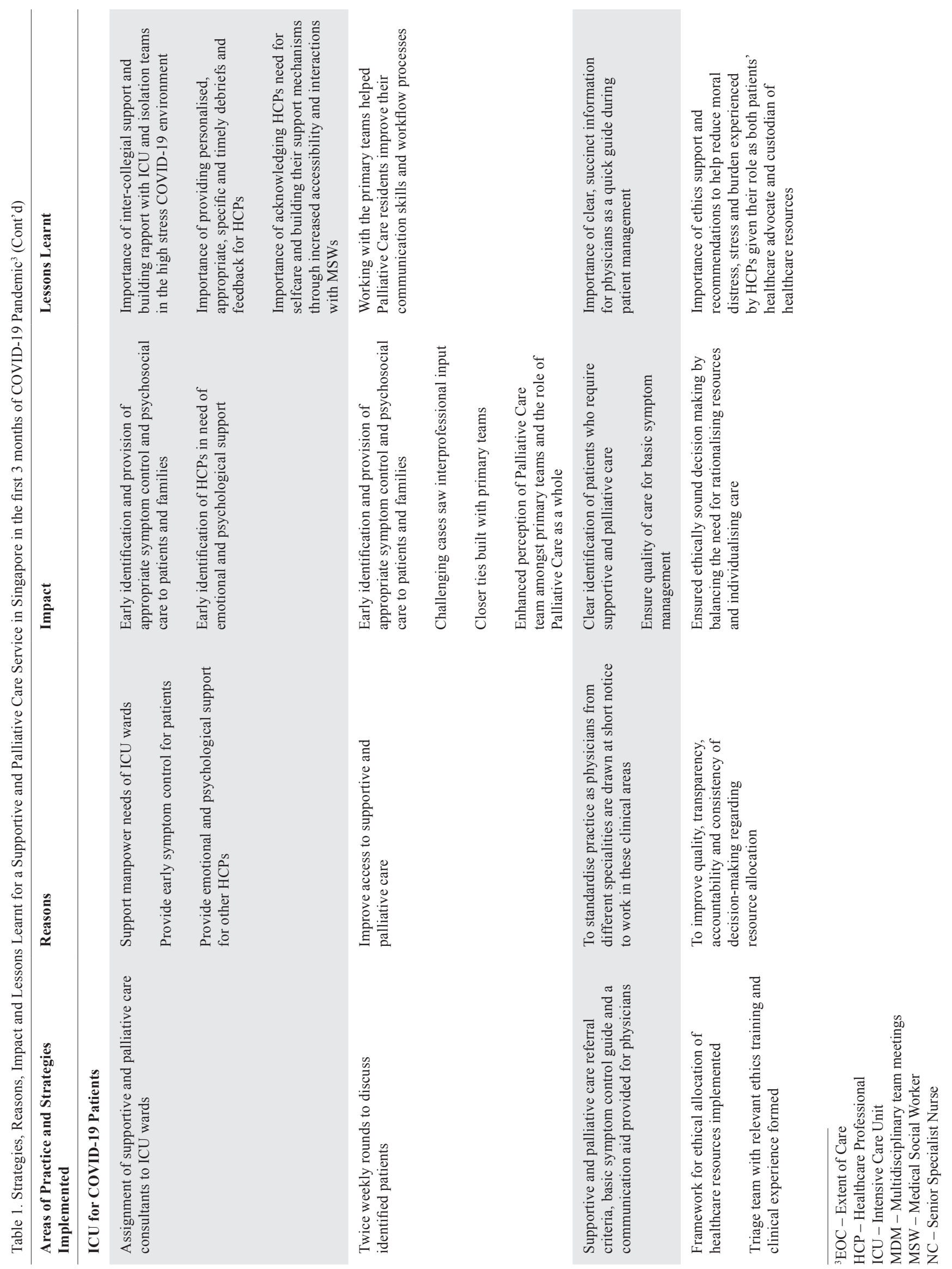




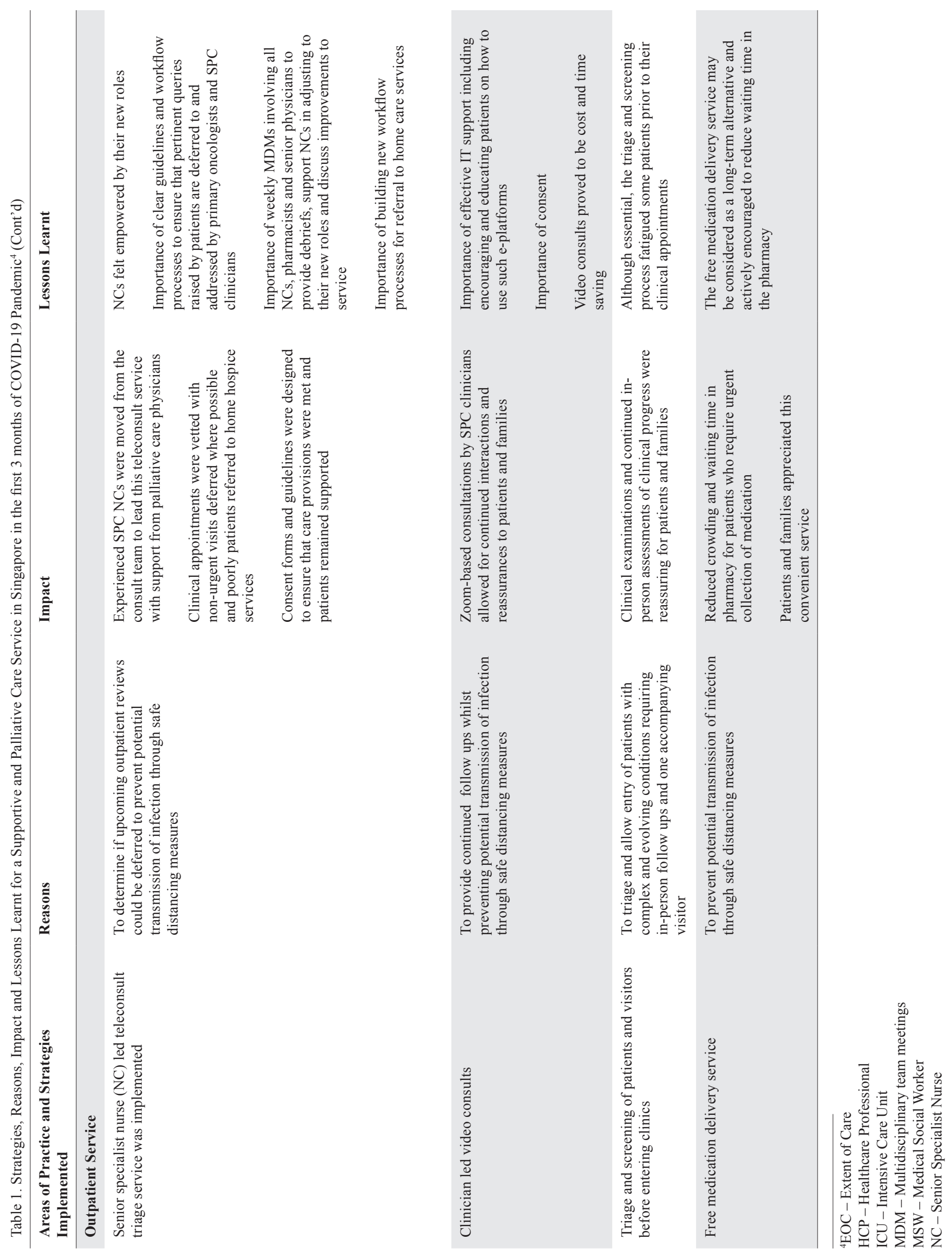


admissions, the small teams had to conserve parenteral medication such as opioids (fentanyl and morphine) and sedatives (midazolam). Fortunately, sublingual and oral preparations for anxiety and agitation, and fentanyl patches or oral morphine for pain control provided reasonable alternatives. ${ }^{6}$

\section{ICU for COVID-19 Patients}

Senior NCCS DSPC physicians and residents were also redeployed to the ICU and isolation wards to provide medical care for COVID-19 patients. The team instituted a clear SPC referral criteria, a basic COVID-19 symptom control guide and a 24-hour telephone consult service to provide timely palliative care support. Online COVID-19 crisis response communication toolkits, SPC toolkits with voice annotated presentations and e-books were also further developed. ${ }^{7}$

The DSPC team was also involved in setting up an ethics framework for the allocation of healthcare resources to improve quality, transparency, accountability and consistency of decision-making. They further served as integral members of an ethics and triage team which was established in preparation for a surge in demand for limited ICU beds. ${ }^{8}$

Having the DSPC team embedded within the ICU not only proved successful in the early identification and provision of appropriate symptom control and psychosocial care to patients and families, but also helped assuage psychosocial, ethical and moral concerns amongst HCPs. Indeed, these paved the way for the establishment of a new SPC-ICU consult service and a recognised referral system.

\section{Outpatient Service}

To enhance social distancing and attenuate risks of infection, senior specialist nurses (NCs) supported by palliative physicians carried out telephone consults for pre-existing SPC patients. The NCs vetted clinical appointments, deferring non-urgent visits where possible and referring poorly patients to home hospice services. A free medication delivery service was also provided and a triage and screening system was set up outside the NCCS building. Outpatient services thus saw a marked $40 \%$ reduction in clinic visits.

The telehealth consults provided by the NC-led team greatly streamlined the delivery of care and influenced the commencement of physician-led teleconferencing. The NCs also notably felt empowered in their new role. However, some observed difficulties in building rapport with patients and noted that reviews were less comprehensive due to time spent addressing logistical issues and anxiety about COVID-19. As such, weekly DSPC MDMs involving all NCs, senior physicians and pharmacists were scheduled to better support the NCs in their provision of effective and targeted care.

\section{Lessons Learnt}

Whilst this adversity has given rise to major changes to SPC services at NCCS and SGH, many lessons have been gleaned in our endeavour to reduce disruptions to our best practices. For one, the quick acclimatisation to and uptake of e-platforms proved vital for the efficacious coordination of care. Their novel use was necessary to facilitate timely information dissemination, continued MDMs and consultations with patients and families. In addition, the importance of upskilling our HCPs rang clear in the face of manpower redeployment and safe distancing measures enforced.

The forging of close interdisciplinary ties between palliative care physicians, residents, nurses and primary teams in the ICU not only enhanced workflow and care delivery processes but also allowed for crucial inter-collegial support. It was pivotal to recognise that the precarious COVID-19 climate posed heightened risks to their personal safety and brought on increased workloads in unfamiliar settings, with challenging decision-making often required of them. To ameliorate distress and fatigue, it was important to offer opportunities for reflection, advice and reprieve through increased access to in-house MSWs and the scheduling of regular team meetings. Instituting clear yet responsive guidelines and protocols was also acknowledged as paramount in alleviating doubts and confusion as HCPs swiftly adjusted to their changing environment.

Through this experience, we have adapted our practice for the safety of all around us and, in so doing, built a sense of camaraderie we had rarely seen before. We hope that our experiences may provide insight to present and future healthcare practices. Indeed even as the crisis abates, it is clear that many of our innovations inspired by this novel pandemic may be here to stay for the betterment of our patients and healthcare professionals alike. 


\section{REFERENCES}

1. Ministry of Health, Singapore. Updates on COVID-19 (Coronavirus Disease 2019) Local Situation. Available at: https://www.moh.gov.sg/ covid-19. Accessed on 10 May 2020.

2. Hsu LY,Chia PY, Lim FY Jeremy. The Novel Coronavirus (SARS-CoV-2) Pandemic. Ann Acad Med Singapore 2020 Mar 16;49(3):105-107.

3. Gov.sg. COVID-19 circuit breaker: heightened safe-distancing measures to reduce movement. Available from: https://www.gov.sg/ article/covid-19-circuit-breaker-heightened-safe-distancing-measuresto-reduce-movement. Accessed on 21 June 2020.

4. Gov.sg. COVID-19 circuit breaker: closure of workplace premises. Available at:https://www.gov.sg/article/covid-19-circuit-breaker-closureof-workplace-premises. Accessed on 21 June 2020.

5. Neo PS, Chan Poon M, Ying Peh T, Ong SY, Hsin Koo W, Santoso U, et al. Improvements in end-of-life care with a protocol-based pathway for cancer patients dying in a Singapore hospital. Ann Acad Med Singapore. 2012 Nov;41(11):483-93.

6. Krishna LKR, Poulose JV, Tan BSA, Goh C. Opioid use amongst cancer patients at the end of life. Ann Acad Med Singapore. 2010;39(10):790-7.

7. Duke-NUS Medical School Lien Centre for Palliative Care. DukeNUS-LCPC COVID-19 Crisis Response Toolkit. Available at: https:// www.duke-nus.edu.sg/lcpc/resources/covid-19-crisis-response-toolkit/ covid-19-crisis-response-communication-toolkit. Accessed on 10 May 2020 .

8. Krishna LKR, Neo HY, Chia EWY, Tay KT, Chan N, Neo PSH, et al. The role of palliative medicine in ICU bed allocation in COVID-19: a joint position statement of the Singapore Hospice Council and the Chapter of Palliative Medicine Physicians. Asian Bioeth Rev 2020 Jun 1:1-7. Available at: https://doi.org/10.1007/s41649-02000128-0. Accessed on 21 June 2020. 